\title{
Coronary arteriovenous fistula originated from left main stem presented by sudden cardiac death
}

\author{
Hyeon Wook Kim and Seung Uk Lee* \\ Division of Cardiology, Kwangju Christian Hospital, Gwangju, Korea
}

\section{Case report}

A 59-year-old man presented to the outpatient clinic of cardiology department with recurrent chest pain for several weeks. An electrocardiogram showed sinus rhythm with $\mathrm{T}$ wave inversion in leads $\mathrm{V}_{2}$ through $\mathrm{V}_{6}$, and appearance suggestive of anterior-wall ischemia (Figure 1). Two-dimensional transthoracic echocardiography showed no regional wall motion abnormality. Elective coronary angiography was performed. Very tortuous and dilated fistula track originated from left main stem linked to 'heart-shaped' small chamber and drained to right atrium on various projection views (Figures 2-4). He was referred for surgical obliteration of the fistula. After surgery, he suffered from postoperative myocardial infarction and heart failure. With ongoing medical conservative treatment, the chest pain and dyspnea resolved within 2 weeks, respectively and discharged. He was discovered with state of death in his home at follow-up 3 months later. The cause of death is not known but suggestive of sudden cardiac death. Perioperative myocardial infarction is an important complication of ligation of coronary artery fistula and could contribute to his sudden death.

Coronary arteriovenous fistulae are a coronary anomaly, presenting in $0.002 \%$ of the general population. The arterial tree can communicate with a cardiac chamber or great vessel in the right side of the circulation (right atrium and its tributaries, coronary sinus, right ventricle, pulmonary artery) causing a left-to-right shunt. The feeding artery of the fistula may drain from a coronary artery or one of its branches and it is usually dilated and tortuous. It is well known that the more proximal the origin from the main coronary artery the more dilated the feeding artery tends to be. Fistulae can be large $(>250 \mathrm{~mm})$ and dilated or ectatic, and they tend to enlarge over time. Therefore, there is a high risk of ischemia in the myocardium beyond the site of the origin of the fistula, which is most frequently evident in association with increased myocardial oxygen demand during exercise or activity. Angina pectoris occurs as a result of the coronary "steal phenomenon" where there is blood shunting and perfusion away from the myocardium. The fistula may rupture spontaneously, causing hemopericardium and cardiac tamponade. Rarely, sudden death can occur. There is general agreement that symptomatic patients should be treated. In addition, closure of fistulae that arise in the proximal segment of the coronary vessel is highly recommended as this type of fistulae is more likely to become aneurysmal with a high possibility of rupture. In this particular case, there are some unique characteristics. First, the fistula track is originated from left main stem, according to my best knowledge this is the first report in the world. Second, tortuous and dilated fistula track linked to small 'heart shaped' chamber and drained right atrium, finally. Third, in the clinical setting, sudden cardiac death occurred at 3 months later after surgery. It is strongly suggestive of relation to postoperative myocardial infarction or spontaneous rupture of ligation site.

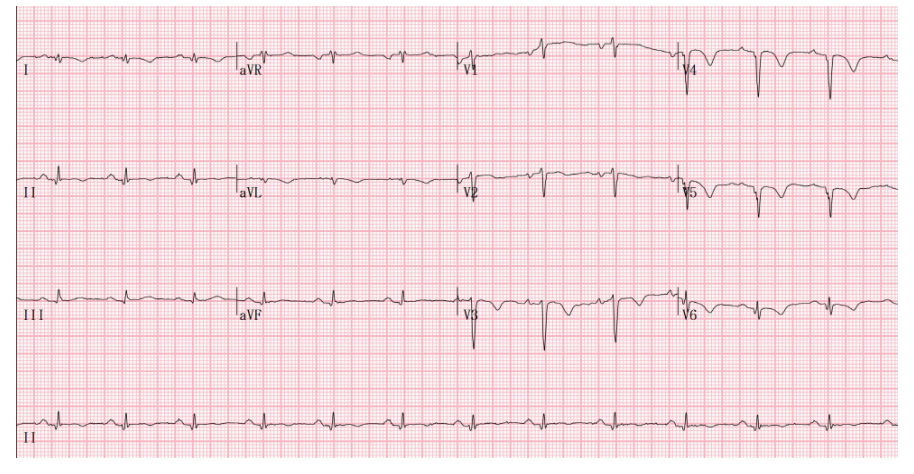

Figure 1. Electrocardiogram

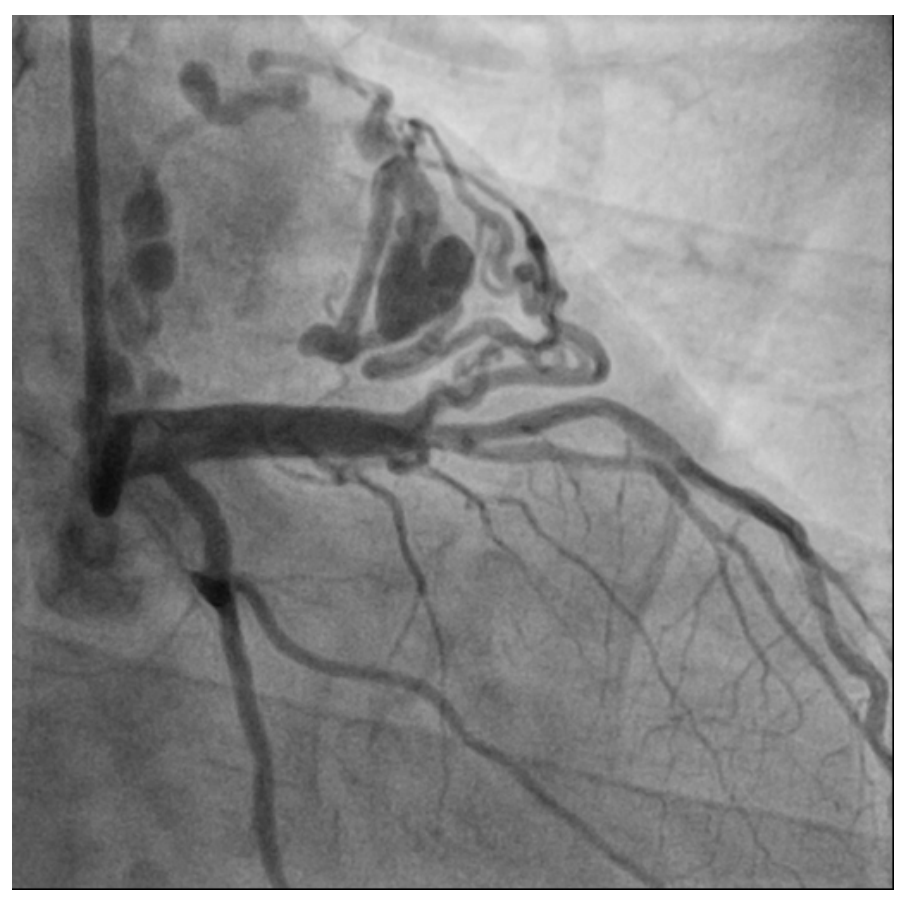

Figure 2. Coronary angiogram with AP caudal view

${ }^{\star}$ Correspondence to: Seung Uk Lee, Division of Cardiology, Kwangju Christian Hospital, Gwangju, Korea, E-mail: cardiosu@hanmail.net

Received: March 01, 2020; Accepted: March 09, 2020; Published: March 13, 2020 


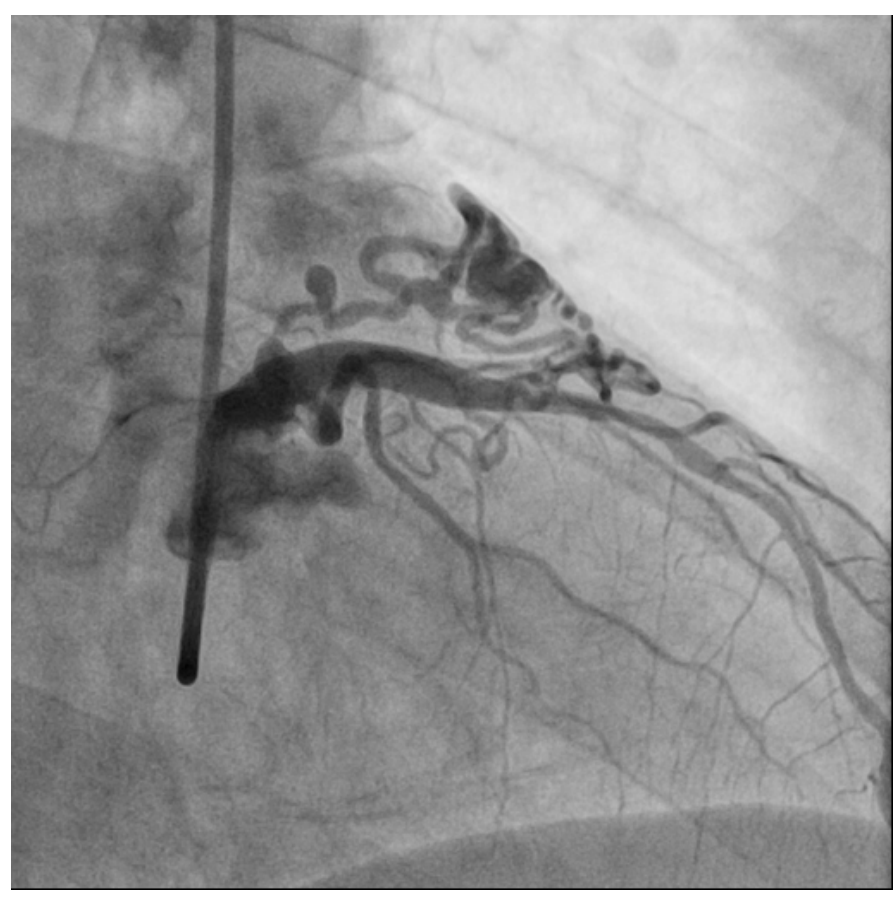

Figure 3. Coronary angiogram with right oblique view

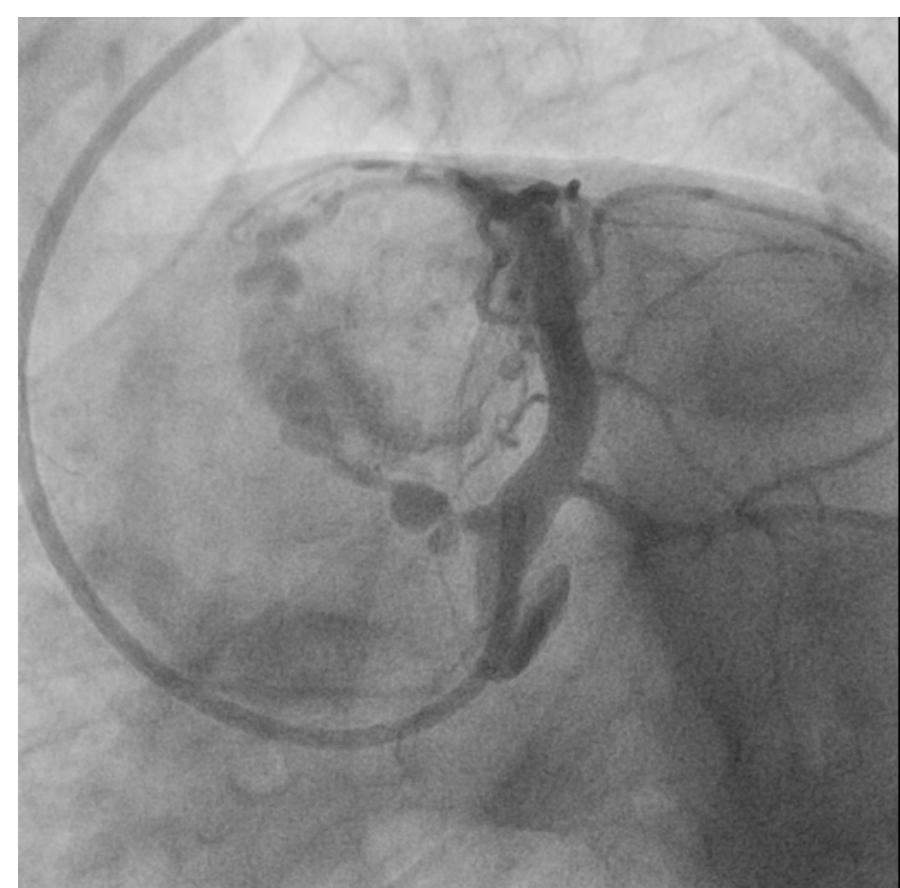

Figure 4. Coronary angiogram with spider view

Copyright: $(2020 \mathrm{Kim}$ HW. This is an open-access article distributed under the terms of the Creative Commons Attribution License, which permits unrestricted use, distribution, and reproduction in any medium, provided the original author and source are credited. 